\title{
Three-dimensional linear stability analysis of cavity flows
}

\author{
Guillaume A. Brès* and Tim Colonius ${ }^{\dagger}$ \\ California Institute of Technology, Pasadena, CA 91125, U.S.A.
}

\begin{abstract}
Numerical Simulations of the two- and three-dimensional linearized Navier-Stokes equations are performed to investigate instabilities of open cavity flows that are homogeneous in the spanwise direction. First, the onset of two-dimensional cavity instability is characterized over a range of Mach numbers, Reynolds numbers and cavity aspect ratios. The resulting oscillations are consistent with the typical Rossiter flow/acoustic resonant modes. We then identify the presence of three-dimensional instabilities of the two-dimensional basic flow and study their dependence on the parameter space. In general, the most amplified three-dimensional mode has a spanwise wavelength scaling with the cavity depth, and a frequency typically an order-of-magnitude smaller than two-dimensional Rossiter modes. The instability appears to arise from a generic centrifugal instability mechanism associated with a large vortex in the two-dimensional basic flow that occupies the downstream portion within the cavity.
\end{abstract}

\section{Introduction}

Open cavities on aircraft are components of a number of systems including weapons bay, landing gear and instrumentation cavities. Self-sustained oscillations inside the cavity generate intense acoustic loading that can lead to structural damage, failure of components, and erratic store separation. In compressible flow, cavity oscillations are typically described as a flow-acoustic resonance involving a feedback process, as shown in figure 1: small disturbances in the shear layer are amplified as they advect downstream through the shear layer and generate acoustic waves upon impingement on the downstream edge of the cavity. These acoustic waves propagate back upstream and interact with the shear layer to excite further instabilities. Resonance occurs at given frequencies, when growing disturbances lead to constructive reinforcement and ultimately saturation. The amplitude of the resulting oscillations is determined by nonlinear effects in the development of the shear layer. This type of flow is referred as shear-layer (or Rossiter ${ }^{1}$ ) mode, and can be distinguished from pure acoustic resonance where frequency selection depends only on acoustic reflections and geometrical parameters. Rossiter performed an extensive set of experiments for two-dimensional rectangular cavities of different length to depth ratio, at different Mach number, which identified a series of discrete frequencies of oscillation. He used the idea of the feedback process to develop a semi-empirical formula to predict the resonant frequencies:

$$
S t_{n}=\frac{f_{n} L}{U}=\frac{n-\alpha}{M+\frac{1}{\kappa}} \quad n=1,2,3 \ldots
$$

where $S t_{n}$ is the Strouhal number corresponding to the $n$-th mode frequency, $f_{n}$. The empirical constants $\kappa$ and $\alpha$ correspond to the average convection speed of the vortical disturbances in the shear layer, and a phase delay (typically $1 / \kappa=1.75$ and $\alpha=0.25$ ), respectively. Data from a large number of experiments and simulations over the years show reasonable agreement with equation 1, but with significant scatter. The scattering is mainly due to the discrepancies between experimental conditions, as different cavity and flow parameters $\left(L / D, L / \Theta_{0}, R e_{\Theta}\right)$ were used. These parameters can have a significant influence on the resonant frequencies and do not appear in Rossiter's formula.

*Ph.D. Candidate, Mechanical Engineering, California Institute of Technology, Pasadena, CA, Student Member AIAA

$\dagger$ Professor, Mechanical Engineering, California Institute of Technology, Pasadena, CA, Member AIAA 


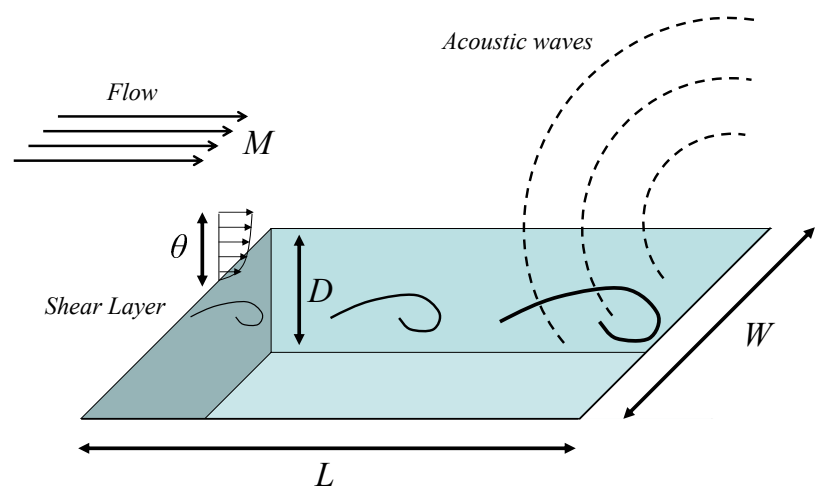

Figure 1. Basic configuration of the cavity flow

Since the earlier work of Rossiter, the basic physics of the two-dimensional cavity instability is now well understood, with reasonanle agreement between experiments and numerical simulations. By comparison, three-dimensional cavity flows have received little attention and remain a challenging subject, but are still highly relevant for practical applications. Recently, some aspects of the 3D cavity flow have been investigated using Large Eddy Simulation (LES) methods (Larchevêque, ${ }^{2}$ Rizzetta and Visbal ${ }^{3}$ ). However, observations regarding the three-dimensionality of the large-scale turbulent structures has not figured prominently in these studies. Likewise, three-dimensional experimental data is limited but several researchers have reported interesting observations of three-dimensionality in cavity flows. Ahuja and Mendoza ${ }^{4}$ conducted an extensive set of experiments on the effect of cavity dimensions, boundary layer and temperature on cavity noise, for subsonic flows with turbulent boundary layer. They reported on three-dimensionality in the mean flow, caused by spillage, flowing over the spanwise sidewalls. Three-dimensional flow features have been observed for wide cavities in early experiments at low subsonic velocities by Maull and East. ${ }^{5}$ Using oil flow visualization of surface streamlines at the bottom of the cavity and surface static-pressure distributions, they showed the existence, under certain conditions, of nearly steady spanwise cellular pattern within the cavity. Rockwell and Knisely ${ }^{6}$ also observed three-dimensional pattern in a water channel experiment for a wide rectangular cavity with laminar boundary layer upstream. A later water tunnel experiment by Neary and Stephanoff ${ }^{7}$ showed similar three-dimensional flow structures as they observed the presence of a transverse wave on the primary vortex of the cavity. Confirmation of the low frequency of the three-dimensional oscillation we obtain is also suggested by their observations. The physics of those structures is still not fully apprehended but appears to be related to the three-dimensional modes we report in section (III).

\section{Simulation and analysis methods}

\section{A. Numerical algorithms}

Following previous work ${ }^{8}$ on cavity flow, we developed a DNS code to solve the full compressible NavierStokes (NS) equations and study the flow over three-dimensional open cavities. The equations are solved directly, meaning that no turbulence model is used and all the scales of the flow are resolved. The NavierStokes equations are written in conservative form as follow:

$$
\begin{aligned}
\frac{\partial \rho}{\partial t}+\frac{\partial}{\partial x_{j}}\left(\rho u_{j}\right) & =0 \\
\frac{\partial \rho u_{i}}{\partial t}+\frac{\partial}{\partial x_{j}}\left(\rho u_{i} u_{j}+P \delta_{i j}\right) & =\frac{1}{R e} \frac{\partial}{\partial x_{j}}\left(\frac{\partial u_{i}}{\partial x_{j}}+\frac{\partial u_{j}}{\partial x_{i}}-\frac{2}{3} \frac{\partial u_{k}}{\partial x_{k}} \delta_{i j}\right) \\
\frac{\partial e}{\partial t}+\frac{\partial}{\partial x_{j}}\left((e+P) u_{j}\right) & =\frac{1}{R e} \frac{\partial}{\partial x_{j}}\left(u_{i}\left(\frac{\partial u_{i}}{\partial x_{j}}+\frac{\partial u_{j}}{\partial x_{i}}-\frac{2}{3} \frac{\partial u_{k}}{\partial x_{k}} \delta_{i j}\right)\right)+\frac{1}{R e} \frac{1}{P r} \frac{\partial^{2} T}{\partial x_{k} \partial x_{k}}
\end{aligned}
$$


with the equation of state

$$
P=\frac{\gamma-1}{\gamma} \rho T
$$

where $\rho, P$ and $T$ are the density, pressure and temperature, and $u_{i}$ is the velocity in the direction of the Cartesian coordinate $x_{i}$. The energy $e$ is defined by $e=\rho\left(E+|u|^{2} / 2\right)$, where $E$ is the internal energy per unit mass. The usual compressible formulation was used to nondimensionalize these equations, where the superscript $d$ refers to the dimensional quantity, and the subscript $\infty$ denotes the free-stream property.

$$
\begin{aligned}
& \rho=\frac{\rho^{d}}{\rho_{\infty}} \quad P=\frac{P^{d}}{\rho_{\infty} a_{\infty}^{2}} \quad T=\frac{T^{d} c_{p}}{a_{\infty}^{2}} \quad e=\frac{e^{d}}{\rho_{\infty} a_{\infty}^{2}} \\
& u_{i}=\frac{u_{i}^{d}}{a_{\infty}} \quad x_{i}=\frac{x_{i}^{d}}{D} \quad t=\frac{t^{d} a_{\infty}}{D}
\end{aligned}
$$

Here, $\gamma$ is the ratio of specific heats, $c_{p}$ the specific heat at constant pressure, $a$ the speed of sound and $D$ the cavity depth. The Prandtl number and the Reynolds numbers are defined respectively as

$$
\operatorname{Pr}=\frac{c_{p} \mu_{\infty}}{k} \quad R e=\frac{\rho_{\infty} a_{\infty} D}{\mu_{\infty}} \quad R e_{\theta}=\frac{\rho_{\infty} U_{\infty} \theta_{0}}{\mu_{\infty}}
$$

where $k$ is the thermal conductivity, $\mu$ the dynamic viscosity and $\theta_{0}$ the initial boundary layer momentum thickness at the cavity leading edge.

A linearized version of the equations is also implemented: we assume that the flow field $\mathbf{q}=[\rho u, \rho v, \rho w, \rho, e]^{T}$ can be decomposed into $\mathbf{q}=\overline{\mathbf{q}}+\mathbf{q}^{\prime}$, where $\overline{\mathbf{q}}$ is a steady solution of the equations and the perturbation field $\mathbf{q}^{\prime}$ verifies $\mathbf{q}^{\prime} \ll \overline{\mathbf{q}}$. Then the Navier-Stokes equations are linearized about $\overline{\mathbf{q}}$ by neglecting higher order terms in $\mathbf{q}^{\prime}$ to give a first order approximation. The perturbation field now satisfies

$$
\begin{aligned}
\frac{\partial \rho^{\prime}}{\partial t}+\frac{\partial}{\partial x_{j}}\left(\bar{\rho} u_{j}^{\prime}+\rho^{\prime} \bar{u}_{j}\right) & =0 \\
\frac{\partial}{\partial t}\left(\bar{\rho} u_{i}^{\prime}+\rho^{\prime} \bar{u}_{i}\right)+\frac{\partial}{\partial x_{j}}\left(\bar{\rho}\left(\overline{u_{i}} u_{j}^{\prime}+u_{i}^{\prime} \overline{u_{j}}\right)+\rho^{\prime} \bar{u}_{i} \overline{u_{j}}+P^{\prime} \delta_{i j}\right) & =\frac{1}{R e} \frac{\partial}{\partial x_{j}}\left(\frac{\partial u_{i}^{\prime}}{\partial x_{j}}+\frac{\partial u_{j}^{\prime}}{\partial x_{i}}-\frac{2}{3} \frac{\partial u_{k}^{\prime}}{\partial x_{k}} \delta_{i j}\right) \\
\frac{\partial e^{\prime}}{\partial t}+\frac{\partial}{\partial x_{j}}\left((\bar{e}+\bar{P}) u_{j}^{\prime}+\left(e^{\prime}+P^{\prime}\right) \overline{u_{j}}\right)= & \frac{1}{R e} \frac{\partial}{\partial x_{j}}\left(\bar{u}_{i}\left(\frac{\partial u_{i}^{\prime}}{\partial x_{j}}+\frac{\partial u_{j}^{\prime}}{\partial x_{i}}-\frac{2}{3} \frac{\partial u_{k}^{\prime}}{\partial x_{k}} \delta_{i j}\right)\right. \\
& \left.+u_{i}^{\prime}\left(\frac{\partial \bar{u}_{i}}{\partial x_{j}}+\frac{\partial \bar{u}_{j}}{\partial x_{i}}-\frac{2}{3} \frac{\partial \overline{u_{k}}}{\partial x_{k}} \delta_{i j}\right)\right) \\
& +\frac{1}{R e} \frac{1}{\operatorname{Pr}} \frac{\partial^{2} T^{\prime}}{\partial x_{k} \partial x_{k}}
\end{aligned}
$$

The existing DNS code can solve linear or nonlinear NS equations for both 2D or 3D flows. The flow equations are solved on a structured mesh, using well-validated methodology: a sixth-order compact finitedifference scheme for spatial discretization in the $\mathrm{x}$ - and $\mathrm{y}$-direction described in Lele ${ }^{9}$ with a fourth-order Runge-Kutta algorithm for time-marching. In the current version of the DNS code, the cavity is supposed homogeneous (periodic) in the spanwise direction (z-direction) and the derivatives are computed using Fast Fourier Transform (FFT) method with subroutines provided by the FFTW ${ }^{10}$ library. Each spanwise wavenumber is discretized into a stretched Cartesian grid, with clustering of points near the walls and the shear layer in the cavity. For the nonlinear cases in 2D, the initial condition is a laminar flat plate boundary layer along the wall and spanning the cavity, with incompressible Blasius profile and spreading rate. The boundary conditions are non-reflective for the inflow and outflow, no slip and constant temperature $\left(T=T_{\infty}\right)$ at the walls (e.g., Thompson, ${ }^{11}$ Poinsot \& Lele ${ }^{12}$ ). In addition, a buffer zone is implemented at the inflow, outflow and normal computational boundaries to reduce acoustic reflections (Colonius, ${ }^{13}$ Freund ${ }^{14}$ ). The code can handle any type of block geometry (including the rectangular cavity shown in figure 2) and is fully parallelized using Message-Passing Interface (MPI). The cavity configuration and flow conditions are controlled by the following parameters: the cavity aspect ratio $L / D$ and spanwise wavelength $\Lambda / D$, the ratio of the cavity length to the initial boundary layer momentum thickness at the leading edge of the cavity $L / \theta_{0}$, the Reynolds number $R e_{\theta}$ and the freestream Mach number $M=U / a_{\infty}$. As temperature differences are expected to remain small, the transport properties are assumed constant: we set $\operatorname{Pr}=0.7$ and $\gamma=1.4$, the values for air. 


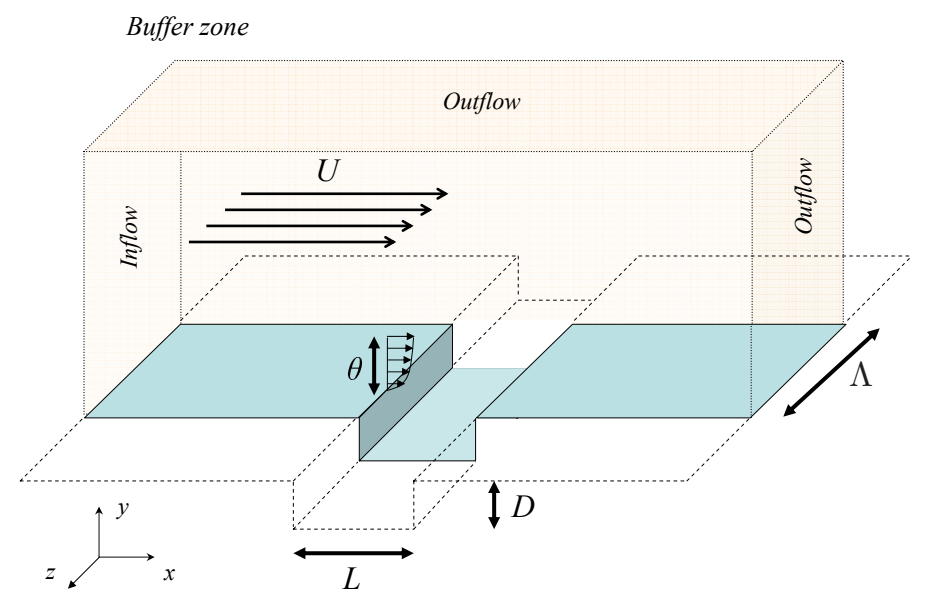

Figure 2. Schematic diagram of the computational domain

\section{B. Linear stability}

In analyzing the behavior of the shear layer oscillations, most investigators have implicitly assumed that the shear layer behavior can be described in isolation, i.e. as if it were a free shear layer. Recent refinements to this model ${ }^{15,16}$ have developed linear theory that couples the shear layer dynamics and acoustic behavior of the cavity (essentially using an unsteady Kutta condition at the cavity leading and trailing edges), but nonparallel shear layer effects and, in particular, the coupling of the flow inside the cavity have not been studied. An alternative analysis of the global instability modes that we pursue here is to consider the basic, steady flow as two-dimensional. This viewpoint requires high-fidelity steady flow solutions of the Navier-Stokes equations as input, and then solves a partial-derivative eigenvalue problem for two and three-dimensional instabilities of the basic flow.

The underlying theory and methodology for extracting these bi- and tri-global instabilities are described by Theofilis ${ }^{17}$ in a recent review paper. Early efforts have concentrated on incompressible flows, including backward facing step, lid driven cavities, laminar separation bubbles, etc. A significant accomplishment of the present work has been to extend this effort to compressible flows where in many cases (including the cavity) small amplitude acoustic radiation is an important aspect of the instabilities and must be treated with high-order-accurate numerics in order to avoid spurious oscillations or numerical dissipation of the relevant instabilities. In the so-called bi-global linear stability theory developed for non-parallel flows by Theofilis, ${ }^{18,19}$ the transient solution of the equations of motion $\mathbf{q}=[\rho u, \rho v, \rho w, \rho, e]^{T}$ is decomposed into

$$
\mathbf{q}(x, y, z, t)=\overline{\mathbf{q}}(x, y)+\mathbf{q}^{\prime}(x, y, z, t)
$$

where $\overline{\mathbf{q}}(x, y)$ is the unknown steady two-dimensional basic flow and $\mathbf{q}^{\prime}(x, y, z, t)$ an unsteady three-dimensional perturbation with $\mathbf{q}^{\prime} \ll \overline{\mathbf{q}}$. As the domain is homogeneous in the spanwise direction, a general pertubation can be decomposed into Fourier modes with spanwise wavenumbers $\beta$. At linear order, modes with different wavenumber are decoupled and the following eigenmode Ansatz can be introduced:

$$
\mathbf{q}^{\prime}(x, y, z, t)=\sum_{n} \hat{\mathbf{q}}_{n}(x, y) \exp \left[i\left(\beta z-\Omega_{n} t\right)\right]+c . c .
$$

where the parameter $\beta$ is taken to be a real and prescribed spanwise wavenumber, related to a spanwise wavelength in the cavity by $\lambda=2 \pi / \beta, \hat{\mathbf{q}}_{n}$ and $\Omega_{n}=\omega_{n}+i \sigma_{n}$ are the unknown complex eigenmodes and corresponding complex eigenvalues, both dependent on $\beta$. Complex conjugation is required in (5) since $\mathbf{q}^{\prime}$ is real. The frequency and the growth/damping rate of the mode are given by $\omega_{n}$ and $\sigma_{n}$, respectively. The long-time behavior of the linear solution will be dictated by the mode with the eigenvalue $\Omega=\omega+i \sigma$ of largest imaginary part, and all the other eigenmodes can then be neglected, provided that the eigenvalues are sufficiently well separated. The flow is said to be sub-critical (stable) if $\sigma$ is strictly negative, neutrally stable if $\sigma=0$, and super-critical (unstable) if $\sigma>0$. 
The 3D linearized equations described in equation (3) can be written symbolically in matrix notation as

$$
\frac{\partial \mathbf{q}^{\prime}}{\partial t}=\mathcal{A}\left(\frac{\partial}{\partial x}, \frac{\partial}{\partial y}, \overline{\mathbf{q}}\right) \mathbf{q}^{\prime},
$$

where $\mathbf{q}^{\prime}$ is the vector of the perturbed conservative variables $\left(\mathbf{q}^{\prime}=\left[(\rho u)^{\prime},(\rho v)^{\prime},(\rho w)^{\prime}, \rho^{\prime}, e^{\prime}\right]^{T}\right)$ and $\mathcal{A}$ is a spatial differential operator depending on the base flow, cavity parameters (aspect ratio, spanwise wavelength, etc.). Once the equations are spatially discretized, we may represent this equation:

$$
\frac{\partial \mathbf{q}^{\prime}}{\partial t}=A \mathbf{q}^{\prime}
$$

where now $\mathbf{q}^{\prime}$ is the discretized solution vector (length $5 N$ where $N$ is the number of grid points), and $A$ is a constant real $5 \mathrm{~N}$ by $5 \mathrm{~N}$ matrix. In this discrete approach, the constant matrix $A$ depends on the (discretized) given steady flow $\overline{\mathbf{q}}$ and the simulation parameters. The stability of this ordinary differential equation depends on the eigensystem of $A$. The eigenvalues $\left(\lambda_{n}, n=1, N\right)$ are $N$ not necessarily distinct solutions of $\operatorname{det}(A-\lambda I)=0$, and the corresponding eigenvectors $x_{n}$ are the linearly independent solutions of $A x_{n}=\lambda_{n} x_{n}$. For the non-defective cases where there are $N$ linearly independent eigenvectors, $X^{-1}=\left[x_{1}, x_{2}, \ldots, x_{N}\right]^{-1}$ exists and the solution can be written symbolically as

$$
\mathbf{q}^{\prime}=e^{A t} \mathbf{q}_{0}^{\prime}
$$

where $\mathbf{q}_{0}^{\prime}$ is the initial perturbation and

$$
e^{A t}=X \operatorname{diag}\left[e^{\lambda_{1} t}, e^{\lambda_{2} t}, \ldots, e^{\lambda_{N} t}\right] X^{-1}
$$

Therefore, for any initial condition, the long-time decay or growth of the solution will be dictated by the eigenvalue $\lambda_{m}$ with the largest real part, and the same conclusion stands if the system is defective. Comparison of equations (5) and (9) reveals that the continuous and discrete formalisms are simply related by $\lambda_{m}=-i \Omega$.

A determination of the least damped (most unstable) modes now amounts to finding the eigenvalues and corresponding eigenvectors of $A$ with the largest real parts (i.e. the largest values of $\sigma$ ). We proceed with two different methods. One is a direct approach, where we directly search for eigenvalues of $A$ using an Arnoldi method developed in the ARPACK software discussed in section C. A second method is to integrate the governing equations directly in the time domain. Provided the least damped modes correspond to distinct eigenvalues, it is possible through long-time integration to isolate the least-damped mode. If that mode is stable, then either the nonlinear or linearized equations could be used to determine the eigenvalue/eigenvector, since for long time all that remains in the nonlinear case is the least damped mode at small amplitude about the steady basic flow. This is in fact the way that our two-dimensional unstable modes are computed, via the so-called residuals algorithm presented in section D.

\section{ARPACK}

A direct tool to study these three-dimensional instabilities is the ARPACK software developed at Rice university. ${ }^{20}$ ARPACK is a collection of Fortran subroutines designed to solve large scale eigenvalue problems and compute a few eigenvalues and corresponding eigenvectors of a general $N$ by $N$ matrix $A$. This is relevant for our study as the 3D linearized equations can be written in matrix notation $\partial \mathbf{q}^{\prime} / \partial t=A \mathbf{q}^{\prime}$, as described in the previous section. Given a cavity configuration and flow conditions, the eigenvalue of $A$ with largest real part (i.e. the least damped or fastest growing 3D mode) could theoretically be directly computed using ARPACK, as well as the corresponding eigenvector to visualize the shape of the instability. In practice, the use of ARPACK was significantly limited by the size and complexity of our problem, as several weeks of computations were required to obtain an eigenvalue and for each case, several runs may be needed to adjust ARPACK parameters. The software package was therefore only used as a validation tool, discussed below in section E.

\section{Residual method and L2 fitting routines}

To determine the least-damped eigenvalue practically, a least-squares fitting method ${ }^{21}$ was applied to the data time history when exponential decay or growth was reached: given the long-time evolution of the vector 
field $q^{\prime}$ at any location $\left(x_{0}, y_{0}, z_{0}\right)$ and an initial guess for the unknown parameters $\overline{\mathbf{q}}\left(x_{0}, y_{0}, z_{0}\right), \hat{\mathbf{q}}_{r}\left(x_{0}, y_{0}, z_{0}\right)$, $\hat{\mathbf{q}}_{i}\left(x_{0}, y_{0}, z_{0}\right), \omega$ and $\sigma$, a set of "best-fit" parameters is computed such that the "merit function" $\chi$, which measures the agreement between the data and the model (with a particular choice of parameters), is minimized. In our case, the model depends nonlinearly on a set $\left(a_{k}, k=1,2, \ldots, M\right)$ of unknown parameters and the "merit function" $\chi$ is defined as

$$
\chi^{2}=\sum_{i=1}^{N_{f i t}}\left[\frac{d a t a_{i}-X\left(a_{1}, a_{2}, \ldots, a_{M}, t_{i}\right)}{\sigma_{i}}\right]^{2}
$$

where $\left(\right.$ data $\left._{i}, t_{i}\right)$ is the set of $N_{f i t}$ data point to fit, $X$ is a fixed function of t and the parameters $a_{k}$, and $\sigma_{i}$ is the measurement error (standard deviation) on the $i$-th data point. Note that since the measurement errors are not known, $\sigma_{i}$ is set to 1 . Following equation (5), the "merit function" takes the form

$$
\chi^{2}=\sum_{i=1}^{N}\left[\mathbf{q}^{\prime}\left(x_{0}, y_{0}, z_{0}, t_{i}\right)-\left(a_{1}+\left(a_{4} \cos a_{2} t_{i}-a_{5} \sin a_{2} t_{i}\right) e^{a_{3} t_{i}}\right]^{2}\right.
$$

Note that the method is sensitive to the initial guess (same order of magnitude than the "best-fit" parameters needed for accurate results) and the length of data to fit (namely if $\mathrm{N}$ is too large and the data still contains transient components, the fit may not be successful). Upon convergence, the mode frequency $\omega=a_{2}$ and growth/damping rate $\sigma=a_{3}$, which are independent of the location $\left(x_{0}, y_{0}, z_{0}\right)$, can be recovered.

With the eigenvalue determined, equation (5) may be written at three different times, $t_{1}, t_{2}=t_{1}+\Delta t$ and $t_{3}=t_{1}+2 \Delta t$ as a linear system of three unknowns $\overline{\mathbf{q}}, \hat{\mathbf{q}}_{r}$ and $\hat{\mathbf{q}}_{i}$. With the transient solution $\mathbf{q}_{n}=\mathbf{q}\left(x, y, z, t_{n}\right)$ available at these times, the system can be solved to deliver the steady-state solution $\overline{\mathbf{q}}$ and the spatial structure $\left(\hat{\mathbf{q}}_{r}, \hat{\mathbf{q}}_{i}\right)$ of the linear eigenmode:

$$
\begin{aligned}
\overline{\mathbf{q}} & =\frac{\mathbf{q}_{1} e^{2 \sigma \Delta t}-2 \mathbf{q}_{2} e^{\sigma \Delta t} \cos \omega \Delta t+\mathbf{q}_{3}}{e^{2 \sigma \Delta t}-2 e^{\sigma \Delta t} \cos \omega \Delta t+1} \\
\hat{\mathbf{q}}_{r} & =\frac{s_{1}\left(\mathbf{q}_{2}-\overline{\mathbf{q}}\right)-s_{2}\left(\mathbf{q}_{1}-\overline{\mathbf{q}}\right)}{c_{2} s_{1}-c_{1} s_{2}} \\
\hat{\mathbf{q}}_{i} & =\frac{c_{1}\left(\mathbf{q}_{2}-\overline{\mathbf{q}}\right)-c_{2}\left(\mathbf{q}_{1}-\overline{\mathbf{q}}\right)}{c_{2} s_{1}-c_{1} s_{2}}
\end{aligned}
$$

where

$$
c_{1}=e^{\sigma t_{1}} \cos \omega t_{1} \quad c_{2}=e^{\sigma t_{2}} \cos \omega t_{2} \quad s_{1}=e^{\sigma t_{1}} \sin \omega t_{1} \quad s_{2}=e^{\sigma t_{2}} \sin \omega t_{2}
$$

\section{E. Validation}

Throughout this study, several test cases were used to validate the method and its implementation. The DNS code was successfully tested through comparison with simple acoustic problems and previous validated numerical results from Rowley et al. ${ }^{8}$ The two-dimensional basic flow calculations were performed on very fine grids (about half a million grid points) and for the cases with a stable $(\beta=0)$-eigenmode, the oscillation frequency was in good agreement with a Rossiter's mode frequency. For the stability analysis, it is particularly important to verify that the modes observed are physical and not generated by any numerical artifact. Several initial conditions at different locations in the cavity were considered to perturb the linear equations and study the flow response. The runs were performed on a coarse grid of 250 by 119 points in the $x-y$ plane. As expected for a global instability, the dominant 3D mode is independent of the initial perturbation: the nondimensionalized frequency and growth rate are identical in all cases, with less than $1 \%$ error. Additionally, to demonstrate grid convergence of the three-dimensional stability computations, the previous simulation was performed on a finer grid of 502 by 239 points in the $x-y$ plane. The structure of the eigenmode is identical on both grid, and the recovery of the eigenvalue leads to the same growth rate and frequency.

Finally, the ARPACK package was used to compute the dominant eigenvalue for the same case (case 2M0325, wavelength $\lambda / D=1$ ). As mentioned in the previous section, the ARPACK software turned out to be fairly inefficient for our study, and was only used here to validate our results. The eigenvalues with the ten largest real parts were computed: as expected, only one mode had an eigenvalue with positive real part, and its frequency and growth rate match the values from the linear stability analysis, again with less than $1 \%$ difference. 


\section{Results}

As the three-dimensional linear stability analysis relies on the existence of a steady two-dimensional basic flow $\overline{\mathbf{q}}(x, y)$, exact solution of the 2D NS equations, the first step is to investigate the stability of twodimensional cavity flows. The theory described in the previous section can be extended to two-dimensional flow by simply setting $w=\bar{w}=w^{\prime} \equiv 0$ and $\beta \equiv 0$ in equations (4) and (5). For a given cavity configuration and flow condition, nonlinear two-dimensional simulations are performed to solve equation (2): as long as the $(\beta=0)$-eigenmode is stable, the basic flow $\overline{\mathbf{q}}$ exists and can be extracted from the DNS data using equation (10), after recovery of the $(\beta=0)$-eigenvalue by the least-squares fitting method. Typical base flows for cavities of aspect ratio 1, 2 and 4 are shown in figure 3 . To conduct the three-dimensional stability analysis, a 3D perturbation of wavelength $\lambda=2 \pi / \beta$ is added to the basic flow $\overline{\mathbf{q}}$, and the linear response $\mathbf{q}^{\prime}$ of the cavity at that particular wavelength is computed by solving equation (3). Linearity reduces the complexity of the full three-dimensional stability problem, as modes with different $\beta$ are decoupled. Several simulations are performed for a discrete set of fixed wavelength $\lambda$ and produced a family of eigenvalue $\Omega_{\lambda}$ and eigenmode $\hat{\mathbf{q}}_{\lambda}$, using again the least-squares fitting method, along with equations (11) and (12). If the two-dimensional flow is unstable then we cannot in principle use DNS to determine the eigensystem since nonlinear effects will become important as the oscillation grows to finite amplitude.

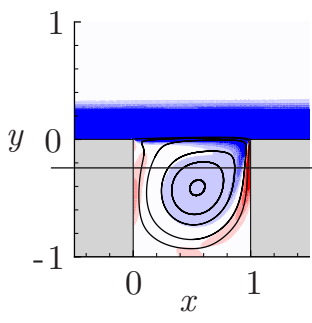

(a)

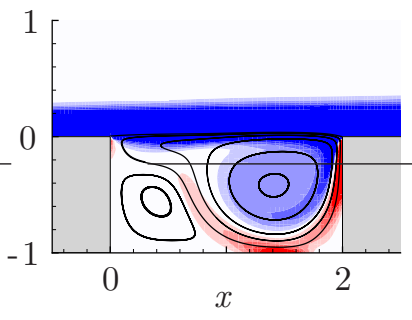

(b)

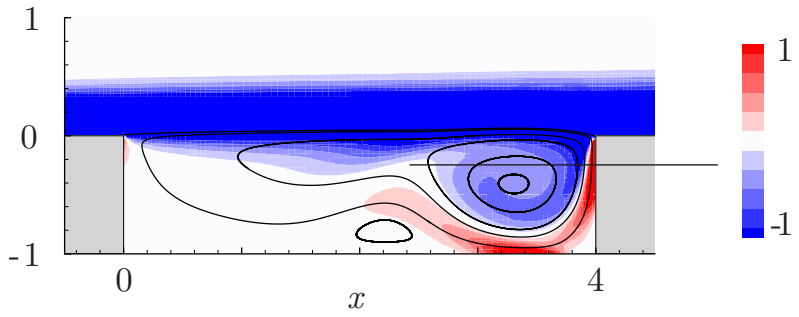

(c)

Figure 3. Vorticity countours and streamlines of the two-dimensional base flow; The vorticity is normalized by the freestream velocity $U$ and ten equally spaced contours between -1 and 1 are shown; (a) run H1Re200; (b) run 2M03; (b) run TK4M03Re65. In each case, the shear-layer and the downstream vortex within the cavity are clearly identified.

\section{A. 2D stability}

In previous work, ${ }^{8}$ supplemented by more recent computations, DNS was used to examine a large portion of the parameter space near the neutral stability curve for the onset of Rossiter mode oscillations. Table 3 provides a summary of runs that are relevant to the discussion that follows. DNS results are classified according to whether the flow was two-dimensionally stable (and thus a steady-state solution was obtained) or whether the flow resulted in self-sustaining oscillations. In many cases we also extracted the growth rate, $\sigma$, frequency $\omega$, and eigenfunction $\hat{\mathbf{q}}$ of the least-damped mode using the methods outlined in section II. In all cases it is observed that the two-dimensional instability is essentially of the Rossiter type, wherein Kelvin-Helmholtz instabilities in the shear layer spanning the cavity are coupled to acoustic feedback and receptivity at the trailing and leading edges, respectively. Frequencies of oscillation are found to be predicted by Rossiter's formula to within the experimental scatter of measurements that have been made over the years. The stability results were used to construct neutral stability curves for two-dimensional instabilities of the basic cavity flows, which are the starting point of the three-dimensional stability analysis. Figure 4 shows a crosssection of the neutral stability curve for two- and three-dimensional instabilities of cavities for runs 2M as a function of the Mach number and Reynolds number. Rowley et al. ${ }^{8}$ previously developped a simple linear model that suffices for predicting parametric variations in the onset of two-dimensional instability. Therefore, our efforts focus on the stability of the three-dimensional modes rather than the Rossiter oscillations. The goal here is to investigate whether or not $3 D$ instabilities take places in the specific region of sub-critical conditions in the parameter space, before the onset of $2 D$ instabilities. Barkley et al. ${ }^{22}$ have used a similar method to show the occurence of a 3D instability within the domain of 2D stability in a backward-facing step. 


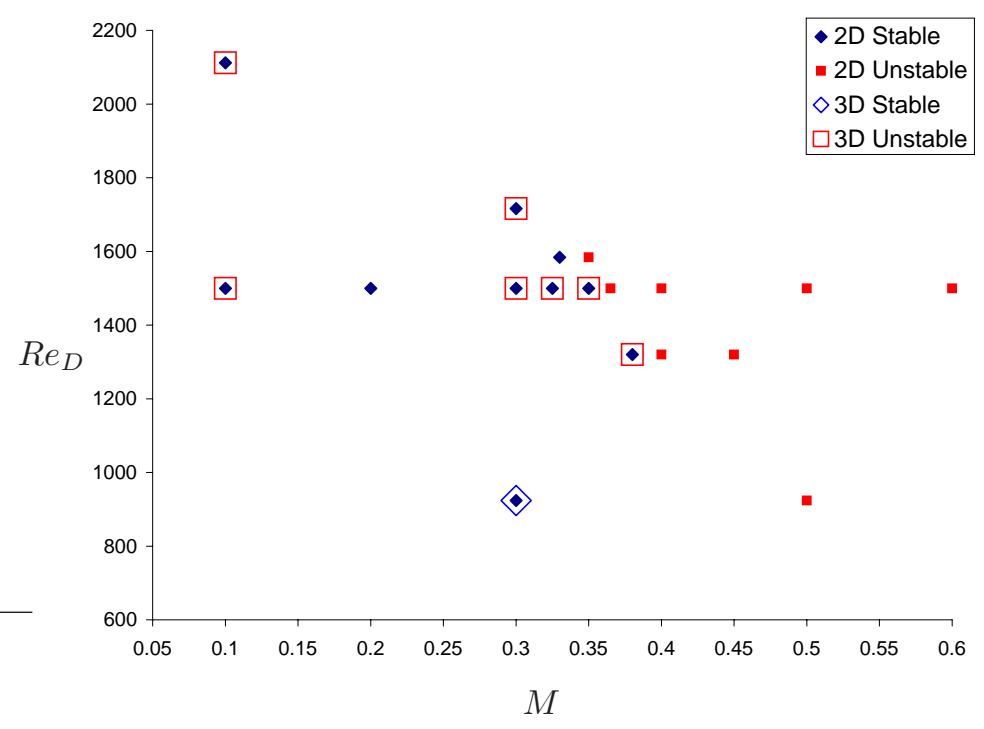

Figure 4. Neutral stability curve for cavity with runs $2 \mathrm{M}\left(L / D=2\right.$ and $\left.L / \Theta_{0}=53\right)$. Both 2D and 3D stability are reported (Note: $R e_{D}=U D / \nu$ ).

\section{B. 3D unstable modes: wavelength and flow structure}

Our linear analysis shows, under most conditions, a single dominant mode in the long-time behavior of the cavity flow for a given spanwise wavelength. For several different cavity configurations and flow conditions, the least damped 3D mode was unstable and exhibited similar features in terms of wavelength and flow structure in all cases. For a band of spanwise wavelength around the size of the cavity depth $(\lambda / D \approx 1)$, an instability would develop in the cavity, which is characterized by a growing disturbance in the base flow vortex that is rotating in the downstream half of the cavity. Figure 5 is a typical time-series showing growth of pertubations. Here, the instability of wavelength $\lambda / D=1$ is growing exponentially while instability of other wavelengths are damped. The flow structure of the unstable mode of spanwise wavelength $\lambda / D=1$ for the same run 2M0325 is shown in figure 6. A cycle of growth and decay of the disturbance can be observed, as it rotates around the primary vortex of the $2 \mathrm{D}$ base flow in the downstream half of the cavity. Starting from the cavity trailing edge, a zone of strong growth exists near the downstream wall where the shear layer impinges the cavity corner. As the fluid in the cavity recirculates upstream and towards the bottom of the cavity, the disturbance amplitude decays slightly, before growing again when moving away from the bottom wall and toward the shear layer. In the shear layer, the perturbation is then significantly damped and convected downstream, until it reaches the cavity corner and starts this cycle again. Overall, a larger growth rate then decay rate in this process leads to reinforcement of the disturbance and ultimately to the three dimensional instability.

\section{Effect of Mach number, Reynolds number and aspect ratio}

Numerous simulations for different cavity aspect ratios and flow conditions were performed, and the results of the three dimensional stability analysis are presented in table 3. As discussed in sections B and D, the dominant eigenvalue $\Omega=\sigma+i \omega$ was computed in each case for a set a discrete spanwise wavelength around $\lambda / D=1$. For several cases, a more extensive set of spanwise wavelengths $(0.25<\lambda / D<16)$ was also considered, but did not lead to any additional instabilities.

The nondimensionalized growth/damping rate $\sigma_{D}=\sigma D / U$ and Strouhal number $S t=\omega D / 2 \pi U$ for the series of runs H1, 2M and TK2 are presented in figures 7, 8 and 9 respectively. Several features of the three dimensional instabilities can be observed. First, the cavity depth $D$ seems to be the appropriate length scale for the three-dimensional instability. The most unstable mode generally occurs for a spanwise wavelength 


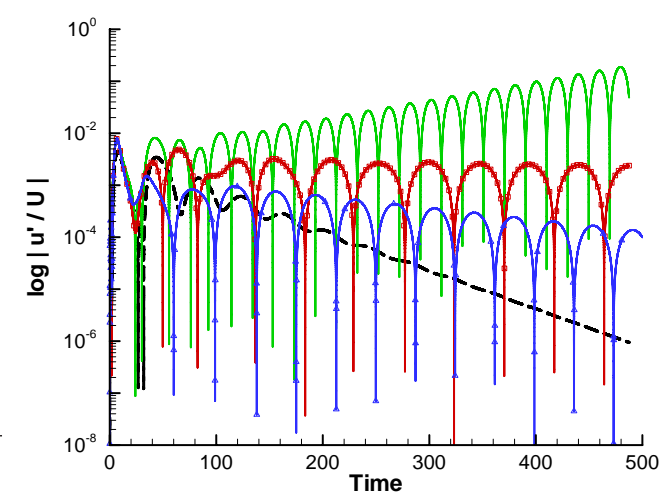

Figure 5. Long-time linear response of the cavity to three-dimensional pertubations of different spanwise wavelengths for run $2 \mathrm{M0325}$ at $(x, y, z)=(L / 2,0,0)$ : (--- ) $\lambda / D=0.5,(-) \lambda / D=1,(\square) \lambda / D=1.5,(\triangle)$ $\lambda / D=2$.

about $\lambda / D \approx 1$. Also, the Strouhal numbers are similar for cavities of aspect ratio $L / D=1, L / D=2$ and $L / D=4$. By contrast, the two-dimensional unstable Rossiter mode has frequency $f L / U$ scaling with the cavity length, $L$. Similarly, the stability transition appears to be dictated by the Reynolds number based on $\mathrm{D}, R e_{D}$, rather than $R e_{\Theta}$. The initial boundary layer momentum thickness $\Theta_{0}$ does not seem to play any significant role in the development of the three dimensional instability (by contrast with the $2 \mathrm{D}$ shear-layer (Rossiter) oscillation).

Another interesting feature is that the three dimensional instabilities have a frequency about an order of magnitude lower than the two dimensional shear-layer (Rossiter) oscillation frequency, which typically range between $f L / U \approx 0.3$ and 1 . Also, The frequency curves in figures 7,8 and 9 tend to suggest that differents modes (at least two) are present. Additional work is required to complete the stability spectrum since the present method can only identified the leading eigenvalue for a given spanwise wavelength.

As observed in particular in figure 9, the Mach number appears to have little influence on the growth rate and frequency of the dominant mode. This feature tends to indicate that the instability is not acoustic but hydrodynamic in nature, as is confirmed by numerical experiments discussed below. On the other hand, the influence of the Reynolds number is significant. In all cases, the growth rate increases with Reynolds number. This results indicates that there must be a critical Reynolds number, above which the flow becomes 3D unstable. Since the Reynolds numbers considered in this study are relatively low, the three dimensional unstable modes are likely to exist for high Reynolds numbers flows in practical applications. Additionally, The frequency decreases with Reynolds number. As discussed in the following section, we found that the mode frequency was strongly connected to the $2 \mathrm{D}$ base flow and its primary vortex within the cavity.

To more fully ascertain the effect of Mach number and Reynolds number on the three-dimensional instability, we perform a set of numerical experiments to assess the differing influence of the basic (steady) two dimensional flow and the Reynolds and Mach numbers. To do this, we construct different cases as follows:

1. Using the methods outlined above, we vary the Reynolds and Mach numbers in both computing the steady base flow (from 2D DNS) and in solving for the three-dimensional disturbances.

2. We artificially increase the Reynolds and/or Mach number acting on the disturbances only, while holding the steady base flow constant. While these simulations are non-physical, they are useful in assessing the flow physics. For the cases where we change the disturbance Reynolds number, the base flow is held strictly constant. For the cases where we change the Mach number, we also rescale the basic cavity flow by assuming that the base flow is essentially incompressible, and rescaling the flow velocity to the higher Mach number.

For example, as the Mach number is varied over the range $0.1<M<0.6$, for a constant spanwise wavelength $\lambda / D=1$, we observe changes in the basic (steady) two-dimensional flow, which lead to different stability results for the three dimensional flow. To separate the Mach number effect from the influence of 

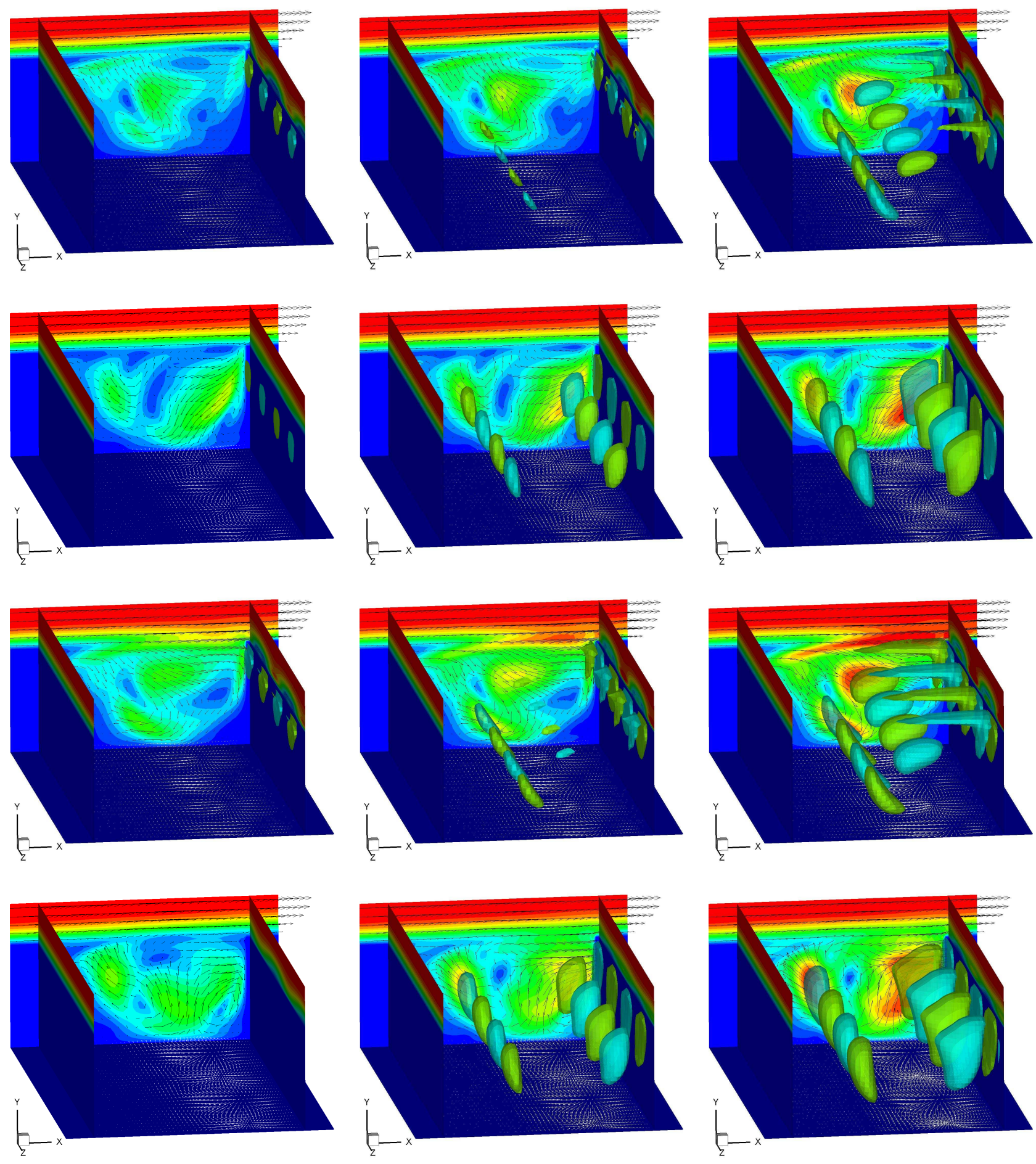

Figure 6. 3D visualization of three periods of the unstable mode of spanwise wavelength $\lambda / D=1$ for run 2M0325 $\left(L / D=2, M=0.325, L / \theta_{0}=52.8, R e_{\theta}=56.8\right)$. For visualization purposes, the perturbation amplitude was increased to approximately $20 \%$ of the freestream amplitude and was added to the basic steady state flow. The color contours represent the total (steady flow and perturbation) velocity magnitude, and velocity vectors are shown on the streamwise cross section at $z=0$ and on the bottom of the cavity. On each plot, two spanwise periods of the unstable mode are shown and the perturbation velocity in the $z$-direction $w^{\prime}$ are represented by the translucent iso-surface. One time period of the instability is shown on each column, with time increasing from top to bottom, and left to right. 


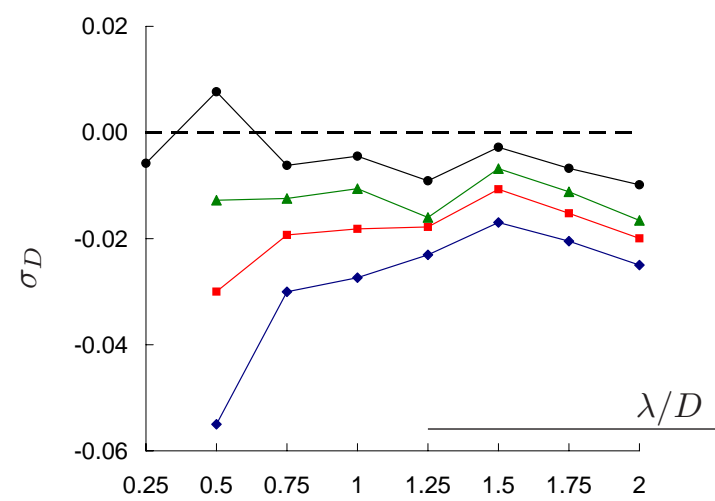

(a)

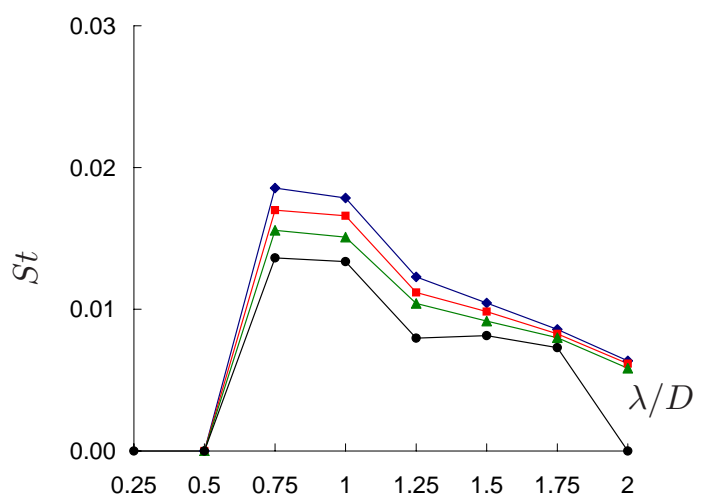

(b)

Figure 7. 3D stability results for runs $\mathrm{H} 1\left(L / D=1, L / \theta_{0}=23.2\right)$. ( $\bullet$ ) H1; ( $\left.\mathbf{0}\right) \mathrm{H} 1 \operatorname{Re} 110 ;(\Delta) \mathrm{H} 1 \operatorname{Re} 140 ;(\bullet)$ H1Re200; The dashed line represents the stability transition $\sigma_{D}=0$; (a) Growth/damping rate; (b) Frequency.

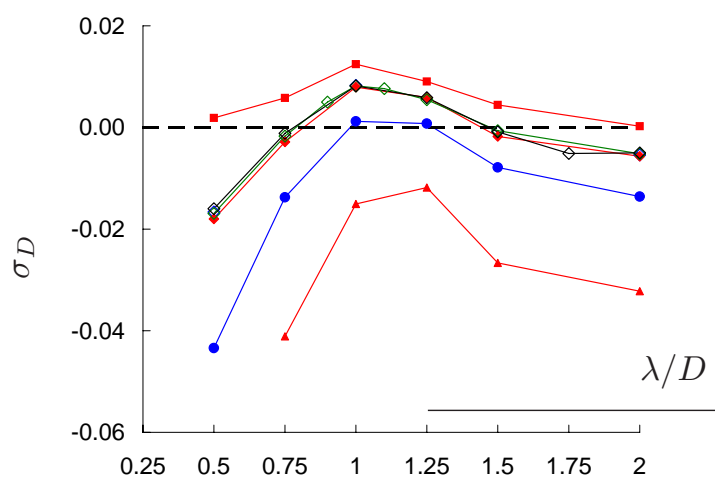

(a)

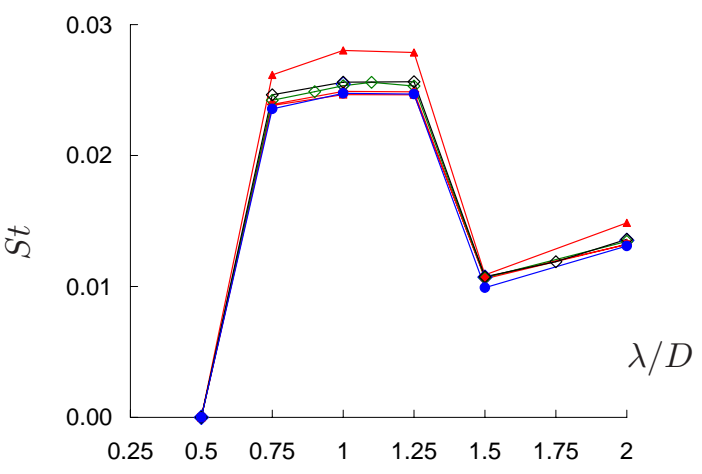

(b)

Figure 8. 3D stability results for runs $2 \mathrm{M}\left(L / D=2, L / \theta_{0}=52.8\right)$. ( $\left.\diamond\right) 2 \mathrm{MO} 1$; ( $\left.\Delta\right) 2 \mathrm{M} 03 \mathrm{Re} 35 ;(\diamond) 2 \mathrm{MO} 3$; ( $\left.\square\right)$ 2M03Re65; $(\diamond)$ 2M0325; $(\diamond)$ 2M035; $(\bullet)$ 2M038Re50; The dashed line represents the stability transition $\sigma_{D}=0$; (a) Growth/damping rate; (b) Frequency.

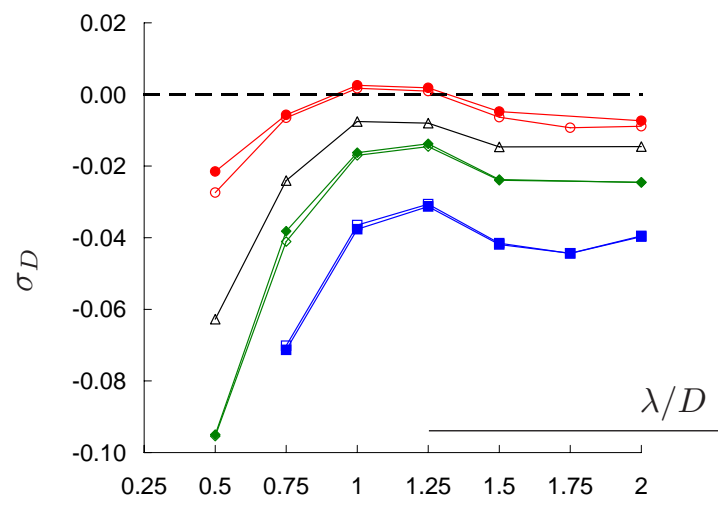

(a)

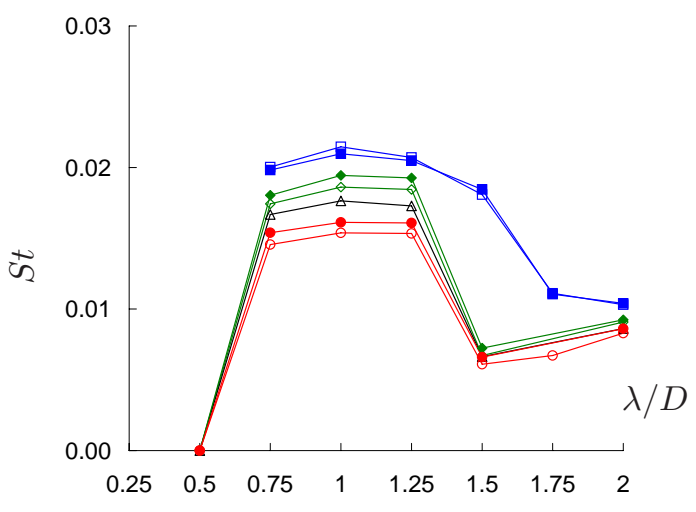

(b)

Figure 9. 3D stability results for runs TK2 $\left(L / D=2, D / \theta_{0}=15\right)$. ( $\left.\square\right)$ TK2M0325; $(\diamond)$ TK2M0325Re80; $(\Delta)$ TK2M0325Re100; ( ○ ) TK2M0325Re140; ( 口 ) TK2M06; ( ) TK2M06Re80; ( • ) TK2M06Re140. The dashed line represents the stability transition $\sigma_{D}=0$; (a) Growth/damping rate; (b) Frequency. 
the basic flow, we performed 3D simulations with a fixed base flow $\overline{\mathbf{q}}=\overline{\mathbf{q}}_{2 M 01}$ obtained for run 2M01 at $M=0.1$. Under the conditions of run 2M01, the flow is 3D unstable, with growth rate and frequency reported in table 1. Before a perturbation at a Mach number $M^{\prime}$ different than $M=0.1$ can be added to the base flow $\overline{\mathbf{q}}_{2 M 01}$, it must be appropriately rescaled to $\overline{\mathbf{q}}_{2 M 01}^{\prime}$ as follow:

$$
\bar{u}^{\prime}=\bar{u} \frac{M^{\prime}}{M} \quad \bar{v}^{\prime}=\bar{v} \frac{M^{\prime}}{M} \quad \bar{\rho}^{\prime}=(\bar{\rho}-1) \frac{M^{\prime}}{M}+1 \quad \bar{P}^{\prime}=\left(\bar{P}-\frac{1}{\gamma}\right) \frac{M^{\prime}}{M}+\frac{1}{\gamma}
$$

The three dimensional stability analysis is then conducted for 3D disturbances at Mach number $M^{\prime}=0.3$ and $M^{\prime}=0.6$, with the corresponding modified base flow $\overline{\mathbf{q}}_{2 M 01}^{\prime}$. These simulations are denoted 2M01toM03 and 2M01toM06 and all the parameters used are summarized in table 1. The comparison with run 2M01 shows that the Mach number has no influence on the three dimensional instability: the flow remained 3D unstable with the results of the rescaled simulations matching the initial run in term of growth rate and frequency, as shown in table 1. A similar parametric study was conducted for $3 \mathrm{D}$ stable flow conditions. The $2 \mathrm{D}$ basic (steady) flow $\overline{\mathbf{q}}_{2 M 03 R e 35}$ of run 2M03Re35 $(M=0.3)$ was modified to get $\overline{\mathbf{q}}_{2 M 03 R e 35}^{\prime}$. Perturbations at $M^{\prime}=0.6$ and $M^{\prime}=0.8$ were then added, for a cavity of spanwise wavelength $\lambda / D=1$. Again the rescaled simulations 2M03Re35toM06 and 2M03Re35toM08 exhibited the same feature than the original run even at these high Mach numbers and remained stable with similar damping rate and frequency than run 2M03Re35. Note that, under all the conditions considered here, the flows at Mach number $M^{\prime}=0.6$ and $M^{\prime}=0.8$ would already be two-dimensional unstable.

\begin{tabular}{ccccccccc}
\hline & \multicolumn{3}{c}{ 2D parameters } & & $\begin{array}{c}\text { 2D base } \\
\text { flow }\end{array}$ & $\begin{array}{c}\text { 3D parameter } \\
\text { modified }\end{array}$ & \multicolumn{2}{c}{ Eigenvalue } \\
\hline run & $L / D$ & $M$ & $L / \Theta_{0}$ & $R e_{\Theta}$ & $\overline{\mathbf{q}}$ & $M^{\prime}$ & $\sigma_{D}$ & $S t$ \\
\hline \hline 2M01 & 2 & 0.1 & 52.8 & 56.8 & $\overline{\mathbf{q}}_{2 M 01}$ & & 0.0083 & 0.0255 \\
\hline 2M01toM03 & - & & - & - & $\overline{\mathbf{q}}_{2 M 01}^{\prime}$ & 0.3 & 0.0085 & 0.0254 \\
2M01toM06 & - & & - & - & $\overline{\mathbf{q}}_{2 M 01}^{\prime}$ & 0.6 & 0.0084 & 0.0254 \\
& & & & & & & & \\
2M03Re35 & 2 & 0.3 & 52.8 & 35 & $\overline{\mathbf{q}}_{2 M 03 R e 35}$ & & -0.0151 & 0.0280 \\
\hline 2M03Re35toM06 & - & & - & - & $\overline{\mathbf{q}}_{2 M 03 R e 35}^{\prime}$ & 0.6 & -0.0152 & 0.0280 \\
2M03Re35toM08 & - & & - & - & $\overline{\mathbf{q}}_{2 M 03 R e 35}^{\prime}$ & 0.8 & -0.0155 & 0.0280 \\
\hline
\end{tabular}

Table 1. Parameters and results of the study of the Mach number influence for a cavity of spanwise wavelength $\lambda / D=1$. Here the superscript (') indicates that the $2 \mathrm{D}$ base flow has been rescaled to the modified Mach number $M^{\prime}$

A similar analysis was performed to study the influence of the Reynolds number independently of the effect of the basic (steady) two dimensional flow. Here, a perturbed flow at three different Reynolds number $\left(R e_{\Theta}^{\prime}=65, R e_{\Theta}^{\prime}=50\right.$ and $\left.R e_{\Theta}^{\prime}=35\right)$ was added to the two dimensional base flow $\overline{\mathbf{q}}_{2 M 03}$ obtained for run 2M03 $\left(L / D=2, L / \theta_{0}=52.8, R e_{\Theta}=56.8\right)$, for a cavity of spanwise wavelength $\lambda / D=1$. Rescaling is not needed in this case, and the resulting simulations are denoted 2M03toRe65, 2M03toRe50 and 2M03toRe35. The flow was artificially stabilized/amplified by reducing/increasing the Reynolds number. The growth/damping rates and frequency of the corresponding three-dimensional instabilities are reported in table 2. For those test cases, it is interesting to note that, while the Reynolds number significantly affects the stability of the dominant mode by changing the growth rate, the frequencies remained identical and similar to the original case 2M03. This feature has to be contrasted with the observations from figures 7,8 and 9 that the frequency decreases with Reynolds number, when full 3D linear stability analysis simulation are conducted with the appropriate corresponding $2 \mathrm{D}$ base flow $\overline{\mathbf{q}}$. However, there is no contradiction there. This indicates that the mode frequency is strongly connected to the basic (steady) two dimensional flow $\overline{\mathbf{q}}$ and is only indirectly affected by the Reynold number through the dependence of $\overline{\mathbf{q}}$ on $R e$. To clarify this point, the results from the run 2M03Re35 are compared to the experimental run 2M03toRe35 in table 2. Again, It is important to point out here that the only difference between these two runs is the use of a different 2D base flow $\left(\overline{\mathbf{q}}_{2 M 03 R e 35}\right.$ versus $\left.\overline{\mathbf{q}}_{2 M 03}\right)$ for the three dimensional stability analysis. Both simulation leads to the same stability result: the flow is $3 \mathrm{D}$ stable. The differences in damping rate and frequency is then caused by the difference in 2D base flow. Similar observations can be made with runs 2M03Re65 and 2M03toRe65 in table 2. 
The following conclusions can then be drawn from these numerical experiments: Firstly, the growth/damping rate of the dominant mode is directly driven by the Reynolds number and is, to some lesser extent, dependent of the base flow. Secondly, the Reynolds number influences the mode frequency only through its effect on the two-dimensional base flow $\overline{\mathbf{q}}$. Finally, within the domain of $2 \mathrm{D}$ stability, the three-dimensional mode is essentially independent of the Mach number.

\begin{tabular}{ccccccccc}
\hline & \multicolumn{3}{c}{ 2D parameters } & \multicolumn{2}{c}{$\begin{array}{c}\text { 2D base } \\
\text { flow }\end{array}$} & $\begin{array}{c}\text { 3D parameter } \\
\text { modified }\end{array}$ & \multicolumn{2}{c}{ Eigenvalue } \\
\hline run & $L / D$ & $M$ & $L / \Theta_{0}$ & $R e_{\Theta}$ & $\overline{\mathbf{q}}$ & $R e_{\Theta}^{\prime}$ & $\sigma_{D}$ & $S t$ \\
\hline \hline 2M03Re35 & 2 & 0.3 & 52.8 & 35 & $\overline{\mathbf{q}}_{2 M 03 R e 35}$ & & -0.0151 & 0.0280 \\
2M03 & 2 & 0.3 & 52.8 & 56.8 & $\overline{\mathbf{q}}_{2 M 03}$ & & 0.0079 & 0.0249 \\
2M03Re65 & 2 & 0.3 & 52.8 & 65 & $\overline{\mathbf{q}}_{2 M 03 R e 65}$ & & 0.0124 & 0.0247 \\
\hline 2M03toRe35 & - & - & - & & $\overline{\mathbf{q}}_{2 M 03}$ & 35 & -0.0244 & 0.0252 \\
2M03toRe50 & - & - & - & & $\overline{\mathbf{q}}_{2 M 03}$ & 50 & 0.0007 & 0.0250 \\
2M03toRe65 & - & - & - & & $\overline{\mathbf{q}}_{2 M 03}$ & 65 & 0.0148 & 0.0248 \\
\hline
\end{tabular}

Table 2. Parameters and results of the study of the Reynolds number influence for a cavity of spanwise wavelength $\lambda / D=1$.

\section{Centrifugal instability}

The question of the mechanisms behind the global three dimensional instability observed remains to be addressed. Our results indicates that the primary vortex in the downstream half on the cavity plays a key role in the reinforcement process of the $3 \mathrm{D}$ disturbances. Therefore we argue here that the main mechanism is the centrifugal instability associated with the closed streamlines in the recirculating vortical flow near the downstream cavity wall (see figure 3). The centrifugal instability problem was first considered for swirling inviscid flow by Rayleigh, who derived the now celebrated Rayleigh's circulation criterion for stability (e.g. Drazin \& $\operatorname{Reid}^{23}$ ). The classical Rayleigh centrifugal instability theory was generalized to two dimensional inviscid flow by Bayly. ${ }^{24}$ For the centrifugal instability to occur, a sufficient condition is that the magnitude of the angular velocity decreases outward in some region of the flow with closed streamlines. Barkley et al. ${ }^{22}$ applied this theory to the three dimensional instability they observed in a flow over a backward-facing step, with good agreement. The lid-driven cavity flow problem is another field of study where three-dimensional features and centrifugal instability mechanism have been connected (e.g. Albensoeder et al. ${ }^{25}$ ). Following the notation from Barkley et al., the Rayleigh discriminant is defined as

$$
\eta(x, y)=-\frac{\partial\|\mathbf{r}(x, y) \times \overline{\mathbf{u}}(x, y)\|^{2}}{\partial r}
$$

where $\mathbf{r}(x, y)=\left(x-x_{c}, y-y_{c}\right)$, with $\left(x_{c}, y_{c}\right)$ the center about which the angular velocity is defined, and $r=\|\mathbf{r}(x, y)\|$. The velocity is simply $\overline{\mathbf{u}}(x, y)=(\bar{u}, \bar{v})$. In practice, the center $\left(x_{c}, y_{c}\right)$ was chosen to be the approximate location of the minimum velocity inside the primary rotating vortex in the cavity. The discriminant $\eta$ is actually only weakly dependent on the choice of the center of rotation: computations with $\pm 10 \%$ error on $\left(x_{c}, y_{c}\right)$ lead to similar results. The flow is now centrifugally unstable in the inviscid limit where $\eta>0$.

Figure 10 shows the Rayleigh discriminant for the 2D base flow of the 3D unstable runs 2M03 and TK4M03Re65. The contours represent only the region where $\eta$ is greater than $5 \%$ of its maximum value. Additionally, the streamline are plotted to visualized the flow. The zone where the angular velocity decreases significantly radially corresponds the downstream part of the primary recirculating vortex near the cavity wall. It also corresponds to the region of maximum growth of the disturbance, as discussed is section B. Intuitively, the presence of the downstream and bottom walls forces a rapid decrease in momentum at the outward streamlines. Because of the wall constraints, the fluid is driven in the spanwise direction to form the strutures observed in figure 6 . As the walls are an intrinsic part of all configurations, any cavity flow should be inherently unstable because of the centrifugal instability mechanism, and our simulations do show regions of positive Rayleigh discriminant even for three-dimensional stable cases. However, the centrifugal stability 
criterion is only a sufficient condition for inviscid flow. As the parametric study of the Reynold number effect showed, viscosity plays a key role in the presence of the three-dimensional modes. In general, simulations with a higher $R e$ lead to larger values and broader zones of positive Rayleigh discriminant, and ultimately higher growth rate of the three-dimensional instabilities. Rayleigh's circulation criterion can also be interpreted in terms of circulation along the closed streamlines. In that case, the flow is centrifugally unstable if the circulation magnitude decreases in the outward direction. To validate our results, the circulation on the closed streamlines around the primary vortex were computed as a function of the distance to the center of rotation. Both independent methods lead to the same result: the Rayleigh's circulation criterion indicates that, in the inviscid limit, the flow will develop a three dimensional centrifugal instability in the primary recirculating vortical flow inside the cavity near the downstream wall.

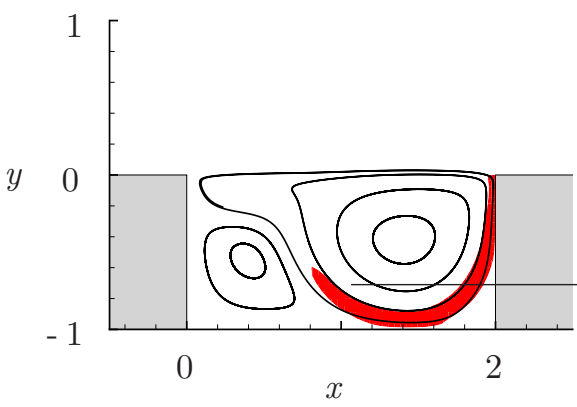

(a)

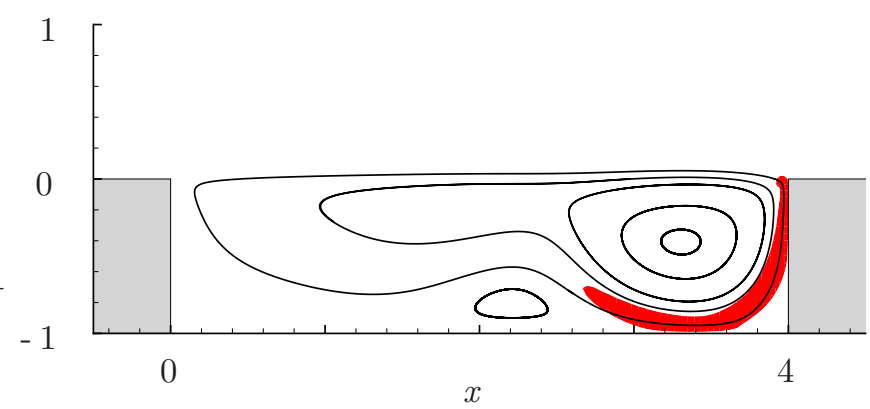

(b)

Figure 10. Streamlines and Rayleigh discriminant of the two-dimensional base flow. Both cases are 3D unstable. The flooded contours shows the region where $\eta$ is greater than $5 \%$ of its maximum value; (a) run 2M03; (b) run TK4M03Re65.

\section{Conclusion}

The three-dimensional stability of open cavities that are homogeneous in the spanwise direction is investigated using the linearized Navier-Stokes equations coupled to eigenvalue/eigenvector extraction algorithms. For sub-critical conditions, we identified, for the first time, the presence of three-dimensional instabilities which take the form of disturbances growing in the $2 \mathrm{D}$ recirculating vortical flow within the cavity. In general, the dominant three-dimensional mode features a spanwise wavelength scaling with the cavity depth, irrespective of the aspect ratio of the cavity, and a frequency about an order of magnitude smaller than for typical two-dimensional shear-layer (Rossiter) oscillations. Through numerical experiments, we showed that the three-dimensional instabilities are unaffected by Mach number over subsonic Mach numbers up to 0.6. It is not expected that they would be significantly altered by compressibility even for cavities with much larger $M$, since they are active within the cavity where the flow speed is much lower than the freestream value. In contrast, the Reynolds number has a significant influence on the mode growth/damping rate and, to some lesser extent, frequency. As the growth rate increases with Reynolds number, the presence of such three-dimensional instabilities is anticipated to be of relevance for higher Reynolds number flow. Additionally, we argue that the three-dimensional modes are connected to a generic centrifugal instability mechanism associated with the primary vortex of the $2 \mathrm{D}$ base flow near the downstream wall. Such vortices are ubiquitous in both the low Reynolds number flows considered here and also (in a time-averaged sense) at much higher Reynolds numbers. Similar mechanism has been suggested for three-dimensional instabilities in flows over backward-facing step ${ }^{22}$ and lid-driven cavity ${ }^{25}$ flows.

Future work includes extension of the linear analysis to super-critical conditions, three-dimensional simulations of the full Navier-Stokes equations to investigate nonlinear effects, and cross-validation of the present results with Large Eddy Simulation or experimental data. It is our hope that the results presented here will help future work on cavity control, as it appears that there may be an untapped potential to exploit inherently three-dimensional effects in cavity flows. 


\section{Appendix}

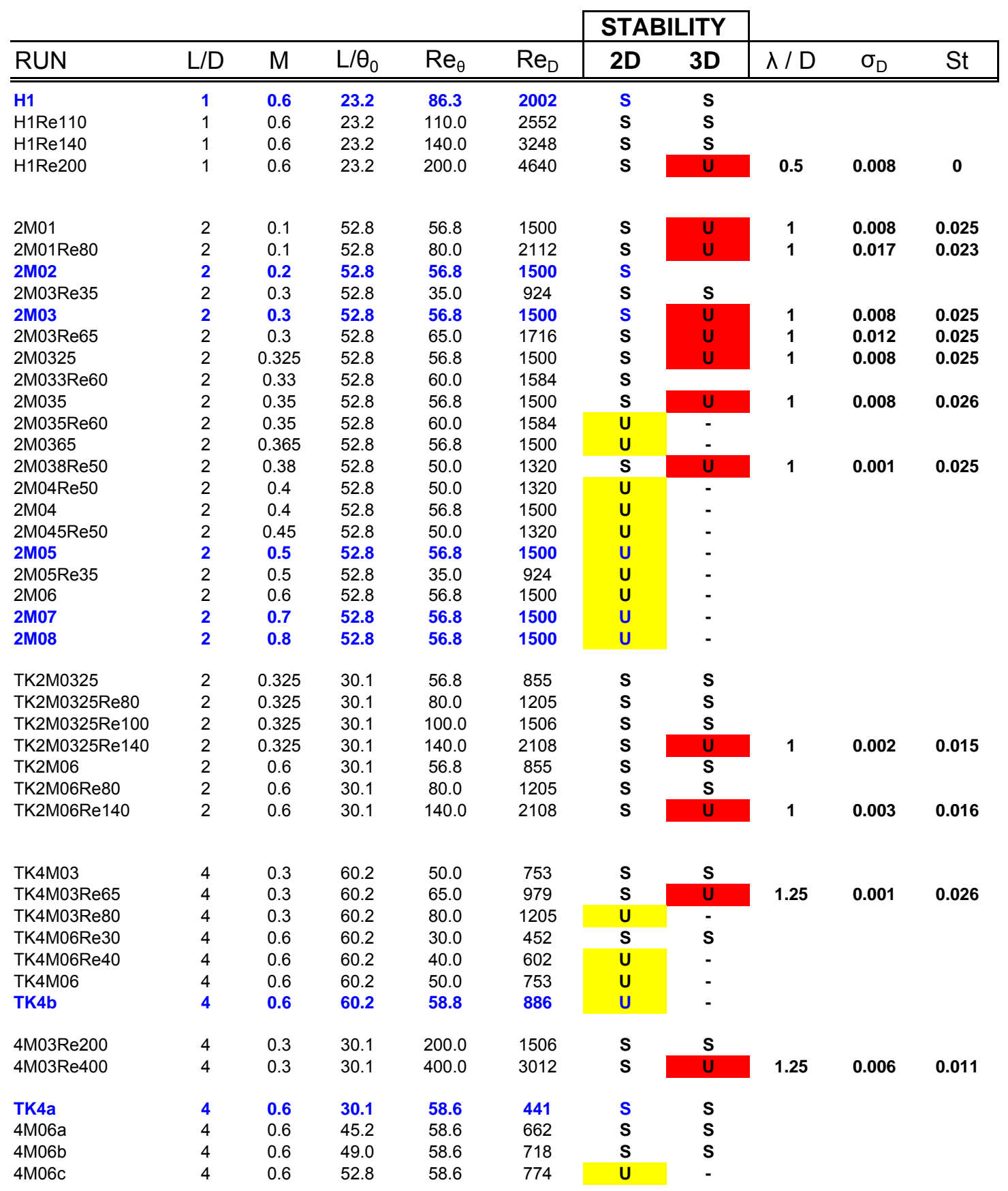

Table 3. Parameters and stability of the different simulations (Note: $\operatorname{Re}_{D}=U D / \nu$ ). Abbreviations for stability are: $\mathrm{S}=$ Stable, $\mathrm{U}=$ Unstable. Run: results from previous work of Rowley et al. ${ }^{8}$

\section{Acknowledgments}

This work was supported by AFOSR under grant F49620-02-1-0362. Computer time was provided in part by the Department of Defense High Performance Computing Centers. Part of this work was done in collaboration with Pr. V. Theofilis from the School of Aeronautics, Universidad Politecnica de Madrid. 


\section{References}

${ }^{1}$ Rossiter, J. E., "Wind-Tunnel Experiments on the Flow over Rectangular Cavities at Subsonic and Transonic Speeds," Aeronautical Research Council Reports and Memoranda, No. 3438, Oct. 1964.

${ }^{2}$ Larchevêque, L., Sagaut, P., Lê, T., and Comte, P., "Large-eddy simulation of a compressible flow in a three-dimensional open cavity at high Reynolds number," J. Fluid Mech., Vol. 516, 2004, pp. 265-301.

${ }^{3}$ Rizzetta, D. P. and Visbal, M. R., "Large-eddy simulation of supersonic cavity flowfields including flow control," AIAA J., Vol. 41-8, 2003, pp. 1452-1462.

${ }^{4}$ Ahuja, K. K. and Mendoza, J., "Effects of cavity dimensions, boundary layer and temperature on cavity noise with emphasis on benchmark data to validate computational aeroacoustic codes," Tech. Rep. CR-4653, NASA, 1995.

${ }^{5}$ Maull, D. J. and East, L. F., "Three-dimensional flow in cavities," J. Fluid Mech., Vol. 16, 1963, pp. 620-632.

${ }^{6}$ Rockwell, D. and Knisely, C., "Observations of the three-dimensional nature of unstable flow past a cavity," Phys. Fluids, Vol. 23, No. 3, 1980, pp. 425-431.

${ }^{7}$ Neary, M. and Stephanoff, M., "Shear-layer-driven transition in a rectangular cavity," Phys. Fluids, Vol. 30, No. 10, 1987, pp. 2936-2946.

${ }^{8}$ Rowley, C. W., Colonius, T., and Basu, A., "On self-sustained oscillations in two-dimensional compressible flow over rectangular cavities," J. Fluid Mech., Vol. 455, 2002, pp. 315-346.

${ }^{9}$ Lele, S. K., "Compact finite difference scheme with spectral-like resolution," J. Comput. Phys., Vol. 103, 1992 , pp. 16-42.

${ }^{10}$ Frigo, M. and Johnson, S. G., "FFTW Library. Website: http://www.fftw.org," 1997-2006.

${ }^{11}$ Thompson, K. W., "Time-dependent boundary conditions for hyperbolic systems, II," J. Comput. Phys., Vol. 89, 1990, pp. 439-461.

${ }^{12}$ Poinsot, T. J. and Lele, S. K., "Boundary conditions for direct simulations of compressible viscous flows," J. Comput. Phys., Vol. 101, 1992, pp. 104-129.

${ }^{13}$ Colonius, T., Lele, S. K., and Moin, P., "Boundary conditions for direct computation of aerodynamic sound," AIAA J., Vol. 31(9), 1993, pp. 1547-1582.

${ }^{14}$ Freund, J., "Proposed inflow/outflow boundary condition for direct computation od aerodynamic sound," AIAA J., Vol. 35(4), 1997, pp. 740-742.

${ }^{15}$ Alvarez, J., Kerschen, E., and Tumin, A., "A theoretical model for cavity acoustic resonances in subsonic flow," AIAA Paper 2004-2845, 2004. 2005.

${ }^{16}$ Alvarez, J. and Kerschen, E., "Influence of Wind Tunnel Walls on Cavity Acoustic Resonances," AIAA Paper 2005-2804,

${ }^{17}$ Theofilis, V., "Advances in global linear instability of non-parallel and three-dimensional flows," Progress in Aerospace Sciences, Vol. 4, 2003, pp. 249-315.

${ }^{18}$ Theofilis, V. and Colonius, T., "An algorithm for the recovery of 2- and 3-D biglobal instabilities of compressible flow over 2-D open cavities," AIAA J., Vol. 2003-4143, 2003.

${ }^{19}$ Theofilis, V., Duck, P. W., and Owen, J., "Viscous linear stability analysis of rectangular duct and cavity flows," J. Fluid Mech., Vol. 505, 2004, pp. 249-286.

${ }^{20}$ Lehoucq, R., Maschhoff, K., Sorensen, D., and Yang, C., "ARPACK software. Website: http://www.caam.rice.edu/software/ARPACK/," 1996-2006.

${ }^{21}$ Press, W. H., Teukolsky, S. A., Vetterling, W. T., and Flannery, B. P., Numerical Recipes in Fortran (second edition), Cambridge University Press, New York, 1992.

${ }^{22}$ Barkley, D., .Gomes, G. M., and Henderson, R. D., "Three-dimensional instability in flow over a backward-facing step," J. Fluid Mech., Vol. 473, 2002, pp. 167-190.

${ }^{23}$ Drazin, P. G. and Reid, W. H., Hydrodynamic stability, Cambridge, New York, 1981.

${ }^{24}$ Bayly, B. J., "Three-dimensional centrifugal-type instabilities in inviscid two-dimensional flows," Phys. Fluids, Vol. 31, No. 1, 1988, pp. 56-64.

${ }^{25}$ Albensoeder, S., Kuhlmann, H. C., and Rath, H. J., "Three-dimensional centrifugal-flow instabilities in the lid-drivencavity problem," Phys. Fluids, Vol. 13, No. 1, 2001, pp. 121-135. 